Association for Information Systems AIS Electronic Library (AISeL)

Wirtschaftsinformatik Proceedings 2005

Wirtschaftsinformatik

February 2005

\title{
Individualising M-Commerce Services by Semantic User Situation Modelling
}

Stefan Figge

Johann Wolfgang Goethe-Universität Frankfurt a. M.

Andreas Albers

Johann Wolfgang Goethe-Universität Frankfurt a. M.

Follow this and additional works at: http://aisel.aisnet.org/wi2005

\section{Recommended Citation}

Figge, Stefan and Albers, Andreas, "Individualising M-Commerce Services by Semantic User Situation Modelling" (2005). Wirtschaftsinformatik Proceedings 2005. 83.

http://aisel.aisnet.org/wi2005/83

This material is brought to you by the Wirtschaftsinformatik at AIS Electronic Library (AISeL). It has been accepted for inclusion in Wirtschaftsinformatik Proceedings 2005 by an authorized administrator of AIS Electronic Library (AISeL). For more information, please contact elibrary@aisnet.org. 
In: Ferstl, Otto K, u.a. (Hg) 2005. Wirtschaftsinformatik 2005: eEconomy, eGovernment, eSociety; 7. Internationale Tagung Wirtschaftsinformatik 2005. Heidelberg: Physica-Verlag

ISBN: 3-7908-1574-8

(C) Physica-Verlag Heidelberg 2005 


\title{
Individualising M-Commerce Services by Semantic User Situation Modelling
}

\author{
Stefan Figge, Andreas Albers \\ Johann Wolfgang Goethe-Universität Frankfurt a. M.
}

\begin{abstract}
The following paper aims at providing a new implementation approach for the individualisation of M-Commerce services. Current market situation as well as academic discussion about intermediaries in the Internet economy will provide the plot for an infrastructure proposal that enables the semantic description of the mobile user's situation to finally unleash the full potential of the mobile usage context. To proof technical feasibility, concepts offered by current ITstandards such as the Semantic Web, Web Services, J2EE and .NET will be assembled to an implementation scenario to demonstrate how individualisation can take place in the fragmented value network of M-Commerce.
\end{abstract}

Keywords: M-Commerce, Intermediaries, Semantic Web, Individualisation

\section{Introduction}

With high-bandwidth mobile networks and powerful mobile devices the technical prerequisites for the provisioning of advanced mobile services are provided. In the application domain of private consumption and commerce this now allows comprehensive support for the satisfaction of consumer needs [Reic +02$]$. The unexpected slow adoption of these so called M-Commerce services combined with high investments that have been made in respective infrastructures were resulting in economic problems within the mobile telecommunications industry [Schw +02 ]. Research on the topic of individualising mobile services is therefore an important facet of finding remedy for this economic problem by improving acceptance and adoption of mobile services.

The open question is, why, even though the positive influence of individualisation on user acceptance is commonly accepted and well-known, its implementation in M-Commerce services has hardly taken place yet. Looking at current mobile portals and services (e. g. Vodafone live!, t-zones or Orange World) only minimal individualisation features, such as approximate positioning or rendering according to the used mobile device, are provided. Comprehensive personal and user specific information, for instance personal preferences, interests or the personal attitude towards the current spatial context, is not taken into account. 
One reason might be that individualisation relies on sophisticated customer and service modelling. To individualise mobile services, the implications of mobile user characteristics on a service's behaviour have to be defined. Additionally, the modelling of the user and the service has to take place within the same semantics and meta-model to become automatically processable. The semantic integration between the customer and service model in the field of M-Commerce is a problem, as the customer model is normally provided by the Mobile Network Operator (MNO) (which "owns" the mobile customer) and the product model by the Service Provider (which provides the product, i. e. the service).

The problem of semantic gaps and disintegration is also an issue in the area of the fixed Internet. It is addressed by the W3C Consortium with its Semantic Web activity [W3C01]. It provides tools and concepts to share semantics across distributed resources and market players. This paper applies these concepts on the mentioned problem of modelling mobile users and their usage situations in order to provide individualised M-Commerce services.

The paper is structured as follows: The next section is discussing mobile services as offerings within economic value chains. Section 3 covers the individualisation aspect and presents how the Semantic Web could contribute. Section 4 is looking at implementation and integration aspects of mobile service offerings by looking at available IT-frameworks. Section 5 is incorporating the introduced concepts within an implementation scenario while section 6 is concluding the paper and provides an outlook on future research.

\section{Mobile Service Offerings in M-Commerce}

It has only recently happened that MNOs opened their mobile data infrastructure to third parties, referred to as Service Providers, for the sake of decentralised and specialised M-Commerce service offerings (e. g. mobile transaction or information services). Now mobile service portals are offered to Service Providers as gates to the huge market shaped by mobile users. For instance, with its currently more than one billion roaming GSM subscribers [GSMA04], MNOs are able to provide access and contact to the majority of the population in the developed countries.

Mobile service portals in that context constitute a semi-open market place controlled by MNOs in which a provider of a mobile service is legally bound to the MNO by contracts regulating the circumstances under which mobile service provisioning can take place. Within these walled gardens, the MNO's customers are navigated to reliable mobile service offerings, which quality is assured by the MNO. 
The offered M-Commerce services come along in two different shapes, depending on the role they play in the value chain of the Service Provider: Either they relate to the primary creation of value, in which case the mobile service itself is a billable mobile information product or mobile information service (e. g. ring-tones or news-services, cf. Figure 1, arrow A). Or they support an economic transaction (e. g. mobile marketing or customer care services, cf. Figure 1, arrow B) in the information, contract, deliver or after-sales phase [Pico+01, p. 50]. In any case the mobile service relates to an economic transaction in a commerce scenario, which is the reason to refer to these services as M-Commerce service offerings in the following.

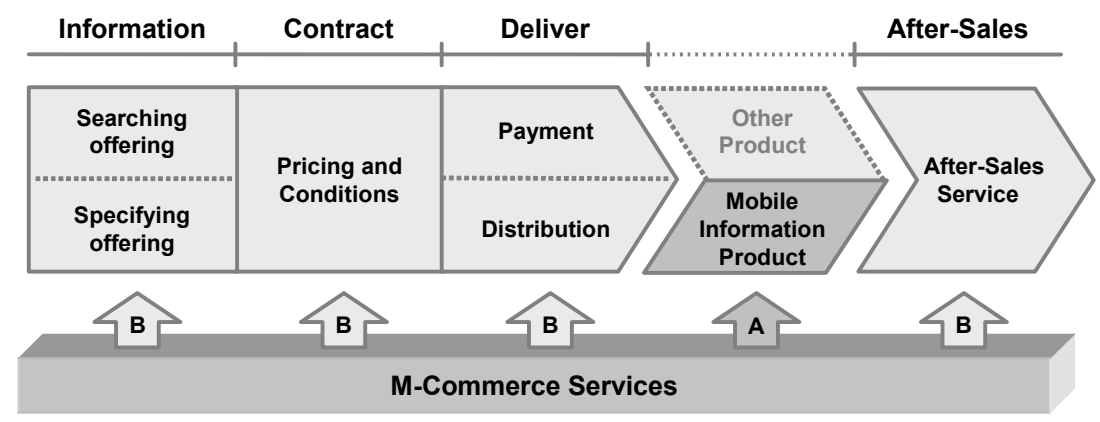

Figure 1: M-Commerce services supporting and fulfilling economic transactions (cf. [Pico+01, p. 50]).

\subsection{MNOs as Intermediaries for Mobile Service Offerings}

By offering mobile service portals, MNOs are responsible for the interface between the Service Provider and the mobile customer. They act as intermediaries in the M-Commerce service domain. In the following it is explained that this mediation simplifies and supports economic transactions through the mobile channel and hence reduces transaction costs for the participating players. For the MNO to take this effort is reasonable to eventually increase traffic and revenues related to the mobile data infrastructure it owns.

Operatively the mediation for the MNO means to aggregate mobile services as well as to operate and to market the mobile service portal [ReMe02]. Aggregating mobile services entails that the MNO is selecting and compositing the kind and number of mobile service offerings that are placed within the walled garden of the mobile service portal. The aggregation task is thereby immediately related to selection, which means that the MNO is evaluating and choosing mobile service offerings according to its set quality requirements. 
Operating the portal requires efficient navigation concepts within the portal. Commonly appropriate portal categories are applied to allow the customer to easily identify service offerings applicable to current needs and problems. It is the individualisation of these portal categories that contributes to a better usability for the user. By reducing mental transaction costs originating from the effort necessary to find a service, the portal appears as a suitable gate to the mobile service market.

Finally, the marketing of the portal is expressed by attracting mobile customers to the provided services by appropriate pricing and access concepts. The application of indirect revenue models for the portal (e. g. by incorporating advertising) as well as easy to use pull- (e. g. a dedicated starting button on the mobile device) and push-access (e. g. by actively informing the user in case of certain events) to the portal are related topics.

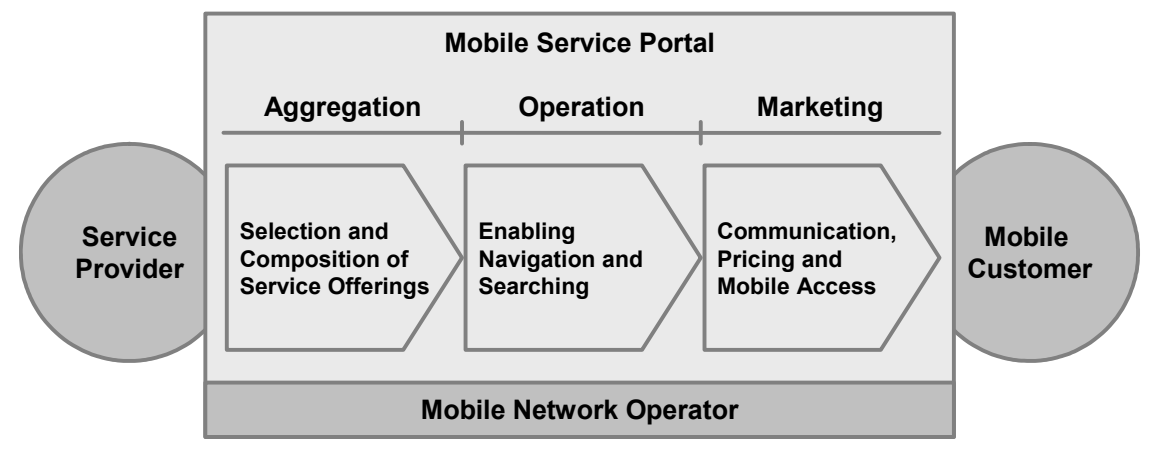

Figure 2: MNO as intermediary between mobile customers and Service Providers

With the mobile service portal, the MNO is continuously resolving an asymmetric distribution of information between Service Providers and mobile customers. The information about identity and position available in mobile networks is aiding the MNO to do so. Individualisation as it is explained in following chapters is applying that ability and enables Service Providers to access semantically described mobile customer relationships through the mobile service portal.

\section{Individualising Mobile Services}

Individualisation means the task necessary to aligning an offering according to the individual preference structure of a user [ReSc02]. Individualisation in the context of this paper can be grasped as the endeavour to adapt a mobile service according to the current individual situation of the user, subsuming his or her personal preferences. From a technical point of view individualisation aims at designing the 
user interface and business logic in such a way that information demands are satisfied with a minimal amount of actions necessary. That contributes to the acceptance and the adoption of the service, as according to IT-acceptance research individualising mobile services is supposed to improve the perceived usability and usefulness [Ambe+03].

\subsection{Implementation Approaches for Individualisation}

There are several alternative concepts to technically implement individualisation. Rule-based, content-based and collaborative filtering are some of the most discussed approaches [KaHa03]. While collaborative filtering relies on the analysis of mass data about past user behaviour, rule-based filtering applies explicitly defined rules and facts that determine the personalised output related to a specific input. These rules and facts partly relate to personal information about the specific users. They can be collected either implicitly by observing the user's behaviour and inferring respective knowledge (e. g. by analysing her or his browsing behaviour) or explicitly by asking the user to disclose the needed information. The implicit approach is related to the problem, that inferred knowledge may be wrong and has to be reconfirmed by the user. Additionally, it is not always transparent for the user on which data the individualisation relies. For these reasons rule based individualisation relying on information explicitly revealed by the customer is applied in the following instead of collaborative or content-based filtering.

From a logic theory perspective rule-based individualisation is based on sets of axioms and inference rules that as clause sets constitute knowledge bases. In the following these clause sets in the sense of description logic will allow to prove theorems about the domain represented as a logical theory [Daco +03$]$. By proving or negating certain theorems, the individualised behaviour of the service is determined.

\subsection{Semantic Customer and Service Modelling}

In order to individualise products and services, information about the customer is necessary. Data available for that purpose constitutes a customer model (e. g. her or his current location, zip-code of the location, attitude towards that location etc.). On the other hand a product or service model, respectively, defines the service behaviour according to different possible customer properties (e. g. the nearest shopping-site for a certain location or special offers for certain customer segments). By matching both models an individual service experience is provided [ScLi02].

A limitation for the individualisation of an M-Commerce service offering is its distributed provisioning which results in disintegrated knowledge and semantics. While the MNO on the one hand has up-to-date information from its network and possibly additional generic and service independent properties obtained from the 
customer (e. g. interests or demographic data), the Service Provider on the other hand may store and manage information specific to its business (e. g. former buying or service specific preferences of the customer).

In order to align and integrate these knowledge bases, a common semantics is necessary. Normally that common semantics is inherently defined by the human actors involved in the individualisation process. By exchanging documents and having implicit notions about concepts, such as location, zip-code or service semantic integration is established manually and coded implicitly within the implementation of the M-Commerce service. By doing so, service offerings are becoming inflexible concerning new features depending on new semantic aspects. Additionally, semantically more complex concepts bear the risk of ambiguity and misunderstandings between the involved parties.

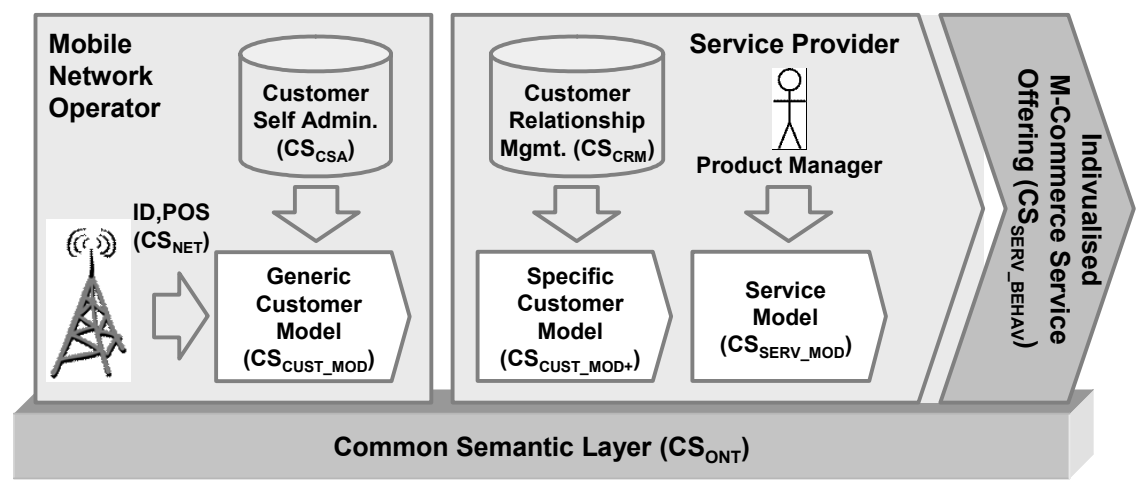

Figure 3: Semantic based individualisation of distributed M-Commerce service offerings

Defining a customer and service model based on an explicit and formally defined common semantics solves these problems as it handles semantics and related knowledge as specific and important design aspects. The ontology concept as it is currently discussed in the area of the Semantic Web (cf. section 4) provides a standardised and well suitable framework for that purpose. First applications can be found in the domain of ubiquitous computing [Chen+03]. Because ontologies as formal semantics definition are applied in the following as the foundation for the customer and service model, these models are subsequently referred to as semantic customer and service models.

\subsubsection{Semantic Customer Model}

The automatic generation of the semantic customer model is enabled by metainformation relating to the mobile communication channel and available in most public mobile cellular networks. As such meta-information, the MNO possesses information about the user's identity and current position (clause set $\mathrm{CS}_{\mathrm{NET}}$ in Fig- 
ure 3). Additionally, the customer may have explicitly disclosed her or his properties and preferences by using customer self-administration applications (clause set $\mathrm{CS}_{\mathrm{CSA}}$ in Figure 3) that most MNOs are nowadays already operating. The MNO is thereby enabled by logic reasoning to generate a service independent generic customer model (clause set $\mathrm{CS}_{\text {CUST_MOD }}$ in Figure 3) describing the current usage situation of the customer by applying individual preferences defined for that situation [Figg +03$]$ (e. g. the user's age, gender, interests or his temporal or geographical context). The information from the mobile network, the customer settings and the common ontology provided by the $\mathrm{MNO}$ (clause set $\mathrm{CS}_{\mathrm{ONT}}$ in Figure 3) can therefore entail the situation description as the generic customer model (clause set $\mathrm{CS}_{\text {CUST_MOD }}$ in Figure 3) offered by the MNO to third parties.

$$
C S_{\mathrm{NET}} \cup C S_{\mathrm{CSA}} \cup C S_{\mathrm{ONT}} \neq C S_{\mathrm{CUST} \_\mathrm{MOD}}
$$

In order to be compliant with current data protection acts [EC02] and to allow the provisioning of the situation description to the Service Provider, it is necessary that the affected user has immediate access and control about her or his personal information (clause set $\mathrm{CS}_{\mathrm{NET}}$ and $\mathrm{CS}_{\mathrm{CSA}}$ in Figure 3). A personal privacy management platform as it is targeted by the European project PRIME [PRIM04] is a necessity to ensure that provisioning of personal information like the situation description is accepted by the user. It could be implemented through the already mentioned customer self-administration applications and should enhance those by adding respective privacy management features.

The Service Provider, provided an already existing relationship with the customer, stores business specific customer information coming from its own customer relationship management databases (clause set $\mathrm{CS}_{\mathrm{CRM}}$ in Figure 3). By incorporating knowledge contained in the generic customer model and the ontology, it is enabled to generate an up-to-date service specific customer model (clause set CS $_{\text {CUST_MOD+ }}$ in Figure 3) describing the customer's current usage situation in the light relevant for the used mobile service.

$$
C S_{\mathrm{CUST}_{-} \mathrm{MOD}} \cup C S_{\mathrm{CRM}} \cup C S_{\mathrm{ONT}} \neq \mathrm{CS}_{\mathrm{CUST}_{\text {_MOD }}+}
$$

\subsubsection{Semantic Service Model}

Based on the structure of the specific customer model and the applied common ontology, the Product Manager of the service can design a service model (clause set $\mathrm{CS}_{\text {SERV MOD }}$ in Figure 3) which states the behaviour and the individualised presentation of the service. By processing the knowledge contained in the customer model, the service model and the agreed ontology, the service behaviour is determined.

$$
C S_{\mathrm{CUST}_{-} \mathrm{MOD}+} \cup C S_{\mathrm{SERV} \_\mathrm{MOD}} \cup C S_{\mathrm{ONT}}=C S_{\mathrm{SERV}_{-} \mathrm{BEHAV}}
$$


The resulting clause set is applied within the service implementation in order to present the service to the customer in a way most appropriate for her or his current usage situation.

\section{Integrative Technologies for Developing Mobile Services}

In order to think about an implementation of the formerly described individualisation approach the following description of IT-concepts shall present the technical toolbox currently available and relevant for that purpose.

\subsection{Remote Procedure Calls and Web Services}

Increasing market dynamics at the end of the last century caused a higher demand for flexibility of organisations from an intra- and inter-organisational perspective. This entailed the need for integration technology that flexibly glued together organisational building blocks for specific businesses purposes. Some of the first technologies serving that objective were remote procedure calls (RPC), objectoriented remote procedure calls (ORPC e. g. DCOM, CORBA or Java RMI) and messaging technologies (MSMQ, MQSeries). The Web Services technology can be grasped as the next generation of RPC [Skon02]. In contrast to former RPC approaches it is based on open, widely adopted standards and focuses on interoperability with the goal to provide a new platform for ubiquitous application integration by using the Internet as its underlying communication platform. Its foundation is XML based messaging over standard web protocols like HTTP or SMTP. The lightweight communication architecture allows any programming language or platform to participate in building distributed applications [Daco +03 ].

The development and deployment of a Web Service can be described as follows: A Web Service provider develops and deploys a Web Service using the programming language and platform of his choice. Then a Web Services Description Language (WSDL) document is created. This document describes the Web Service interface for potential Web Service consumers. The Web Service is finally registered and published in Universal Description, Discovery, and Integration (UDDI) registries. These directories enable Web Service consumers to search for offered services by either querying for technical or business-orientated functionality. If a potential Web Service consumer wants to use a service found in a UDDI registry, its application binds to the Web Service and invokes its methods using Simple Object Access Protocol (SOAP). SOAP defines a XML message format for exchanging data between Web Services and its clients mainly using HTTP as transport protocol [Daco+03]. 


\subsubsection{J2EE and .NET}

Java 2 Platform Enterprise Edition (J2EE) from Sun and the Microsoft.NET platform are currently the two major IT-frameworks providing support for the Web Service technology and therefore enable distributed applications involving several heterogeneous sub-applications.

The important difference between J2EE and .NET is that J2EE is a standard specification supported by many different manufactures, while .NET itself is a product supplied by Microsoft as the exclusive manufacturer. J2EE as a standard only specifies an agreement between applications and containers in which these applications run [Sun04]. As long as an application follows the J2EE specification it is able to run in various container environments on different platforms. The foundation for J2EE is the Java programming language. Applications written in this language are transformed into Java Bytecode, which can be deployed to any platform featuring the Java Runtime Environment (JRE).

In contrast to the J2EE standard the .NET platform encompasses a product framework provided by Microsoft. As one of its most important features the .NET technology offers language-independence and -interoperability. For instance a component written in C\#.NET can be integrated and used into an application written in VB.NET. This is archived by translating the source code into the neutral Microsoft Intermediate Language (MIL). This MIL code, comparable to Java Bytecode, is then executed by the Common Language Runtime (CLR), the equivalent of the JRE [ThLa03].

\subsection{Semantic Web}

While the Web Service technology focuses on integrating distributed business logic in order to constitute an applications, the Semantic Web approach dedicates its efforts in providing "a common framework that allows data to be shared and reused across application, enterprise, and community boundaries" [W3C01]. As an extension to the current Web, it gives information a formally well-defined meaning and makes it machine-discoverable and processable. Figure 4 shows the layers of the Semantic Web architecture.

The lowest Semantic Web layer provides the ability to build resources based on international character sets (Unicode) and to uniquely identify those resources using the Uniform Resource Identifier (URI) standard. On top of that layer, the XML technology including its corresponding XML schema concept is used to create self-descriptive and machine-processable documents. Built upon XML, the Resource Description Framework (RDF) defines a language to add metadata relating to a specific resource in order to describe its contents in a semantic way. Based on this, the ontology vocabulary models the universe of discourse by defining concepts and their relation. It allows defining taxonomies (e. g. class $P D A$ is a 
subclass of class Mobile Device), equivalence relationships (e. g. Cellular Phone equals Mobile Phone) and inference rules to deduct conclusions (e. g. taken three persons $\mathrm{A}, \mathrm{B}, \mathrm{C}$; $\mathrm{A}$ is a relative of $\mathrm{B}$ and $\mathrm{B}$ is a relative of $\mathrm{C}$, hence, $\mathrm{A}$ must also be a relative of $\mathrm{C}$ ) [KoMi04].

The top levels of the Semantic Web architecture consist of the three layers logic, proof and trust. Although these layers are still subject to research their basic purpose is set. The logic and proof layer are supposed to extend the ability to reason and infer knowledge on the foundation of the logic concepts defined by the ontology. Finally, the trust layer is supposed to proof that a certain person or system actually created a document or statement by using digital signatures [KoMi04].

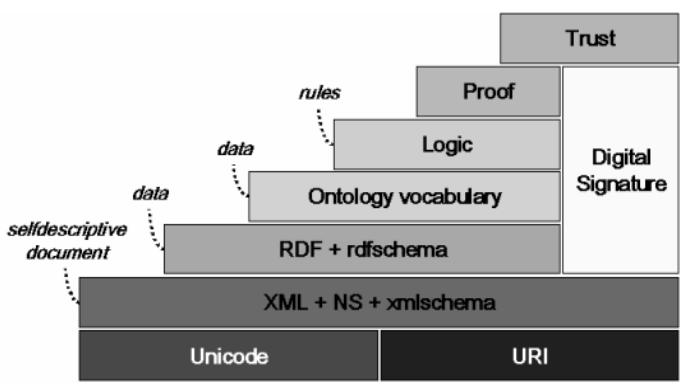

Figure 4: The Semantic Web architectural layers [KoMi04]

As a current field of development the Semantic Web Service approach is building the bridge between the use of distributed business logic (Web Services) and sharing/processing data across domain boundaries (Semantic Web). It is supposed to give applications the ability to automatically discover and integrate services from different providers.

\section{Implementation Scenario for an Individualised M-Commerce Service}

In order to illustrate how Semantic Web, Web Services, J2EE and .NET play together in order to shape individualised M-Commerce services, an implementation scenario is presented in the following. It describes a business context and sketches a developed prototype. It encompasses a mobile service portal implemented in J2EE and an example M-Commerce service implemented in .NET. They are both integrated using Web Services and Semantic Web concepts. 


\subsection{The Scenario}

A fictitious MNO offers its customers mobile data services based on a browser application installed on the customers' mobile phones. A portal presents the set of available mobile service offerings through the browser application to the customer. The offerings are structured by using portal categories that aid the task of finding appropriate offerings for a certain demand.

One of the many fictitious Service Providers that offers services through the MNO's portal is an airport maintainer (AM) company. It provides services to run an airport infrastructure and its facilities (e. g. airport management, ground handling, flight- and terminal operation, etc.). The AM has two customers or business partners, respectively. On the one hand there are airlines that use the maintained airport as departure and destination for their flights. On the other hand there are passengers that use the airport infrastructure to access flight connections. Due to increasing competition between different airports the AM seeks for new services that support passengers in order to gain a competitive advantage compared to other airports. At the same time the AM does not have comprehensive information about the passengers as those are primarily the customers of the airlines.

In order to aid passengers to reach a certain flight connection, the AM in cooperation with the airlines provides a mobile service called Mobile Airport Travel Support (MATS). It provides features such as detailed maps about the terminals, information about airport facilities etc. A specific feature that is described in the following supports frequent flyers on their way to the airport by continuously evaluating their situation shortly before their flight is leaving. So, for example if they running the risk of missing their flight (e. g. due to heavy traffic) they are receiving suggestions what to do best in order to still reach the flight with minimum effort and risk. A prerequisite to use that MATS service feature is that the customer has formerly subscribed at the AM's stationary website and entered necessary information. Shortly before the travel to the airport the customer initiates the MATS service through the service link placed in the portal category travel assistance at the mobile service portal and indicates that the travel to the airport has started by activating a respective option.

\subsection{Mobile Service Portal}

The mobile service portal is the entry point for the customer to use any MCommerce service offering. The customer starts the portal by pressing a dedicated button on her or his mobile phone. It automatically generates an HTTP request with the URI associated with the portal and sends it through the GSM mobile network. When receiving the HTTP request the Portal Maintainer (PM) Servlet (cf. Figure 5) requests from the Situation Maintainer (SM) Servlet (cf. Figure 5) the generation of a situation description for the current mobile user. The SM is calling 
subsequently the Situation Determination (SD) Servlet (cf. Figure 5) to retrieve the necessary data from the mobile network through a dedicated interface. As response, the SD is provided with the customer's position, identity and the local time (cf. $\mathrm{CS}_{\mathrm{NET}}$ in Figure 3).

With that information available two different knowledge bases are consulted by the Situation Computer (SC) Servlet in order to retrieve a comprehensive picture about the customer's usage situation. The personal knowledge base is constituted by personal preferences and properties that have been formerly disclosed and managed by the customer (cf. $\mathrm{CS}_{\mathrm{CSA}}$ in Figure 3). The general knowledge base is constituted by general (i. e. independent from the specific customer) rules and definitions. The domain ontology as described in chapter 3.2 as well as a geographic information system (GIS), which in the case of the prototype is a MapInfo software, are part of this knowledge base. The response of the SC is a generic cus-

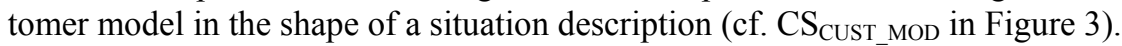

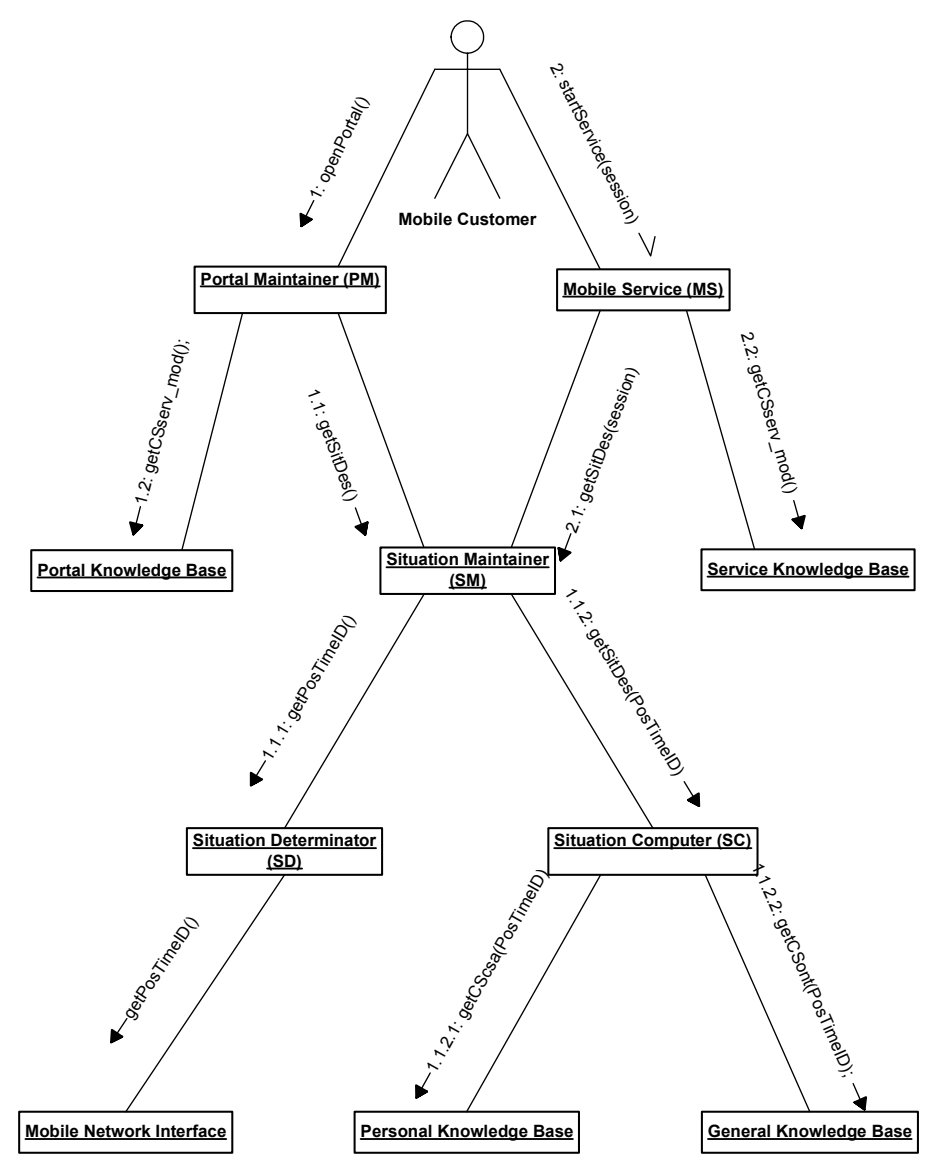

Figure 5: UML collaboration diagram presenting components and sequence of actions 
With the situation description available the PM consults the Portal Knowledge Base which stores the semantic service model (cf. section 3.2.2.). The reason that not only a mobile service but also the portal is associated with a service model is that also the portal can be grasped as a service that can be individualised. In the specific context of the portal that individualisation is expressed by portal categories and service links that are either appearing or not depending on certain values of the situation description. The appearing or hiding of a specific portal object is determined by performing logic reasoning using knowledge bases coded in Prolog. A Java-to-Prolog interface allows the portal to perform queries by using the situation description as a parameter. The result of a specific query finally determines the appearance of a portal category or service link.

By that the PM presents an individualised mobile service portal to the customer who then chooses the mobile service appropriate to her or his current demands. By selecting the MATS service from the portal category travel assistance, the mobile device opens an HTTP requests with the URI related to the MATS service and thereby redirects the browser to the Web-Application constituting the mobile service of the AM. The session ID of current session of the customer is added as a parameter to the HTTP request to allow the MATS service to access the formerly generated situation description for the customer.

\subsection{Portal Interface}

After the MATS service implemented in .NET has received the HTTP request, the service subsequently requests the situation description from the MNO. It is doing so by calling a function that is revealed by the MNO as a Web Service and that demands the provided session ID and the Service Provider ID of the AM. The first is needed to achieve the reference with the customer's session; the second identifies the Service Provider which influences the information that it is going to be included in the situation description that it receives.

This selective provisioning of information is controlled by the customer, who with the help of the customer self-administration functionality can define which information is allowed to be transferred to which Service Provider. The need for such identity management features becomes obvious when talking about the inclusion of attributes which reveal the identity of the customer to the Service Provider.

For instance assuming that the airline British Airways is a Service Provider in the MNO's portal and the customer participates in the airline's customer loyalty program, she or he would have been provided with a respective customer loyalty ID. In order to experience a single-sign-on functionality in the mobile context, the customer might want to provide British Airways with this already assigned customer loyalty ID. This would enable British Airways to generate a service specific customer model as it allows mapping the mobile usage situation to own service specific data such as past flights or the current number the bonus miles acquired. 
In terms of anonymity, pseudonymity and identity [HaPf03] the provisioned customer loyalty ID as a pseudonym provides the Service Provider with the missing link to resolve the formerly anonymous customer relationship. It identifies the customer within the anonymity set of the airline's customers. For that reason, the customer would insist on that any identifying attribute is only revealed to Service Providers which she or he has previously authorised. It is the task of the portal interface together with the customer self-administration to ensure this identity management functionality in order to be compliant with current privacy and security legislation.

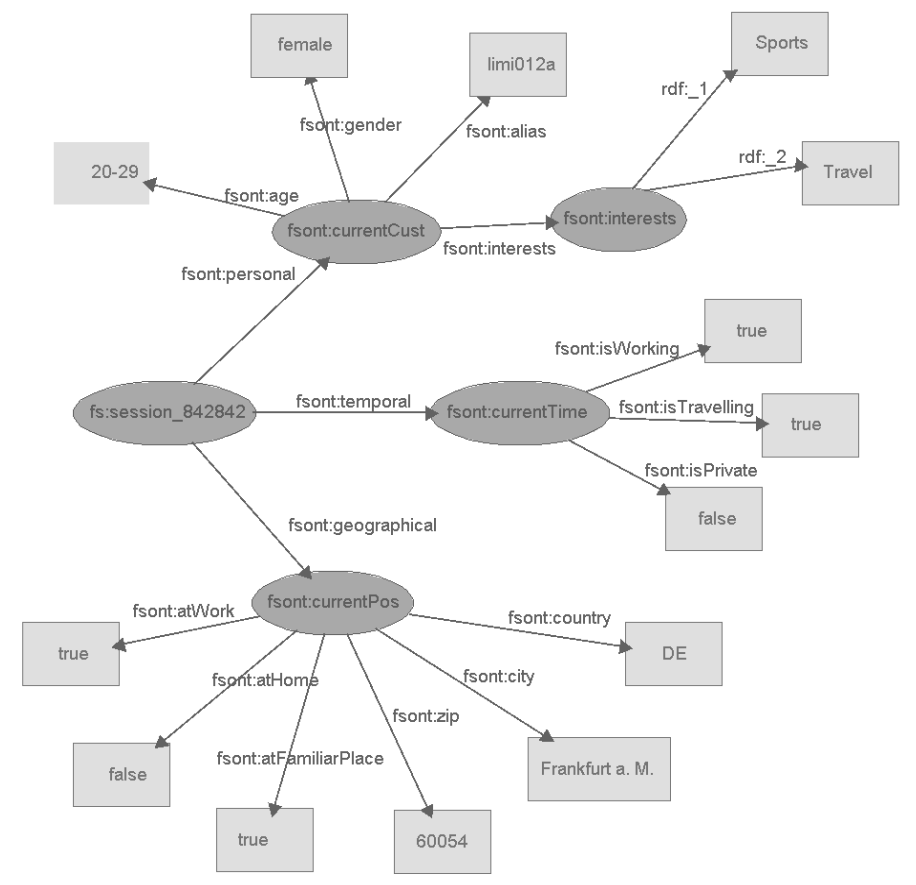

Figure 6: A sample RDF situation description as generic customer model (visualised using the IsaViz tool to be downloaded from www.w3.org/2001/11/IsaViz/)

Figure 6 illustrates a potential situation description that as a generic customer model the MNO is providing as a document coded in RDF to the AM. In terms of the Semantic Web, the situation description relates to the RDF-tier in the Semantic Web stack (cf. Figure 4) and provides knowledge about the current customer and also allows a mapping to existing service specific information. Based on the service specific customer information, the service model and the defined ontology (relating to the ontology-tier in the Semantic Web stack, cf. Figure 4), the Service Provider can define rules (relating to the rules-tier in the Semantic Web stack, cf. Figure 4) that define the individualisation. 
The situation description in Figure 6 shows the information related to the current usage situation which is referenced using a session ID. The situation description is consituted by three branches which describe personal, geographical and temporal aspects. For example, the personal branch states that associated alias or pseudonym, respectivly, is "limi012a" and it also reveals the age and the gender of the current user. The geographical branch states the current country, city and zip-code as well as the personal attitude of the customer towards that position. In this case it is stated that the customer is currently located at her or his working site and not at home and that she or he is familiar with the current location. In the temporal branch it is stated that the customer is currently not in a private temporal context but working. Additionally, it is stated that he or she is currently supposed to be travelling. All branches are related to a certain ontology namespace (fsont) that would formally explain the semantics of each of the used concepts such as age, atWork or isPrivate.

\subsection{Mobile Service}

After the customer has selected the MATS service from the portal, the HTTP request had triggered the Microsoft Internet Information Service (MIIS) at the AM and called the portal interface implemented by the MNO as a Web Service (cf. section 5.3). Now, having obtained the situation description as the generic customer model by using the session ID, the MATS displays an ASP.NET based welcome screen. This welcome screen could already present individualised features based solely on that generic customer model. For instance, the service could offer detailed maps about the airport if the customer is in a travelling related temporal context, already very close to the airport and not familiar with the current surroundings. It could also offer travel instructions for public transport if the customer is already quite near to the airport or it could present navigation instructions for arriving by car if the customer is further away.

For a more specific individualisation it is supposed that the customer had primarily entered relevant information such as a flight number, the planned way of travelling to the airport (e. g. by car, train, plane, etc.) and the type of luggage (e. g. hand luggage) at the AM's stationary website. The customer had therefore been provided with a respective alias (the alias "limi012a") that allows the AM to store service specific information about the customer and provides the link to generate a service specific customer model based on the situation description in the mobile usage context.

In this scenario, the customer tries to catch a flight with a certain flight number, travels by car and carries only hand luggage. Once the customer starts travelling to the airport, she or he activates the flight arrival features of the MATS service via the mobile service portal and explicitly approves the tracking of her or his position. By obtaining the alias as the pseudonym under which the preferences have 
been stored, the MATS service resolves the departure time by analysing the flight number and starts to continuously request the customer's situation description from the MNO. This is done by invoking the Web Service from the MNO's portal interface. During the travel to the airport, the customer's position is extracted from the RDF situation description. By using a GIS system, in the case of the prototype it is provided by a routing service from MapInfo, the estimated time of arrival at the airport can be calculated.

Assumed that after a short time of driving the customer runs into heavy traffic causing a significant delay, the MATS service recognises that the customer might be late for the flight and reacts accordingly. As in the scenario the customer is female, travelling by car with only hand luggage, the MATS automatically suggests several services to ensure the arrival of the flight. For instance it offers the reservation of a dedicated female parking space or it could provide a pre-check-in. It could also request an airport shuttle for the transfer from the parking space to the flight gate. The related information informing about the actions that might be taken is displayed through the mobile device to the customer in the car who then has to reconfirm them. Subsequently, the chosen services are booked and the necessary actions are taken.

Up to certain degree of delay, these actions might help the customer to still reach the booked flight. It also provides a unique service experience enabled by information distributed information that is semantically integrated by a respective customer and service model.

\section{Conclusion and Outlook}

The presented ideas demonstrate how the Semantic Web together with current ITstandards could be combined in the context of the M-Commerce domain in order to individualise mobile service offerings. By semantically and technically integrating knowledge about the mobile customer, the Service Provider is able to gain a comprehensive picture about the customer's current situation allowing to provide an improved service experience. Subject of further research in particular are the following topics:

- Analysing the right properties to be included into the situation description to provide a comprehensive picture with a minimal number of properties.

- Evaluating existing standards to be incorporated into the common ontology (e. g. standardised time or space ontologies).

- Applying additional Semantic Web concepts as they are standardised. Candidates are the web ontology language $\mathrm{OWL}$, the rule markup language RuleML and software allowing reasoning based on ontologies. 
- Creating a Semantic Web Service framework to allow MNOs to automatically discover and integrate mobile services into their portal.

Finally, one of the most important research goals is to apply semantic user situation modelling not only to individualise mobile service offerings. The objective is also to redirect value streams in the mobile market. By marketing the attention that mobile customers are paying to their mobile device when they use the mobile service portal, a new business model in the M-Commerce domain is enabled. In times where customer attention is a limited resource, semantically described and attentive contacts to mobile customers are a valuable item. As a consequence, new business opportunities for the MNOs as well as any other mobile data provider appear that represent potential revenue sources necessary to cope with the high investments in data infrastructures made in recent years.

\section{Acknowledgements}

This work is supported by grants from the German Federal Ministry for Education and Research as well as T-Mobile International. The implementation scenario is supported by MapInfo and its data providers TeleAtlas und DDS. The authors gratefully acknowledge this support.

\section{References}

[Ambe+03] Amberg, M.; Wehrmann, J.; Hirschmeier, M.: Ein Modell zur Akzeptanzanalyse für die Entwicklung situationsabhängiger mobiler Dienste im Compass Ansatz. In: Pousttchi, K.; Turowski, K. (Edt.) Mobile Commerce - Anwendungen und Perspektiven, 3rd Workshop on Mobile Commerce (MC3). Köllen: Bonn, 2003, pp. 73-87.

[Chen+03] Chen, H., Finin, T., and Joshi, A.: An Ontology for Context-Aware Pervasive Computing Environments. Presented at the 18th International Joint Conference on Artificial Intelligence, Acapulco, Mexico.

http://ebiquity.umbc.edu/v2.1/_file_directory_/papers/63.pdf, Download 2004-06-01.

[Daco+03] Daconta, M. C.; Obrst, L. J.; Smith, K. T.: The semantic Web: a guide to the future of XML, Web services, and knowledge management. Wiley: Indianapolis, 2003.

[EC02] European Commission: Draft Directive on data protection and privacy. http://europa.eu.int/information_society/topics/telecoms/regulatory/new_rf/index_en.ht m\#dp, 2002, Download 2004-06-01.

[Figg+03] Figge, S.; Schrott, G.; Muntermann, J.; Rannenberg, K.: EARNING M-ONEY A Situation based Approach for Mobile Business Models. In: Proceedings of the 11th European Conference on Information Systems (ECIS). Naples, 2003. 
[HaPf03] Hansen, M.; Pfitzmann, A.: Anonymity, Unobservability, and Pseudonymity - A Proposal for Terminology. http://www.freehaven.net/anonbib/papers/ Anon_Terminology_v0.14.pdf, 2003, Download 2004-06-01.

[GSMA04] GSM Association: GSM Subscriber Statistics. http://www.gsmworld.com/news/statistics/substMATS.shtml, 2004, Download 200406-01.

[KaHa03] Kaspar, C.; Hagenhoff, S.: Individualität und Produktindividualisierung - Kundenprofile für die Personalisierung von digitalen Produkten. Work Report of the Department Wirtschaftsinformatik II, No. 17. Göttingen University: Göttingen, 2003.

[KoMi04] Koivunen, M; Miller, E.: Semantic Web Activity. http://www.w3.org/2001/12/semweb-fin/w3csw, Download 2004-06-07.

[Pico+01] Picot, A.; Reichwald, R.; Wigand, R. T.: Die grenzenlose Unternehmung. Gabler: Wiesbaden, 2001.

[PRIM04] PRIME: Privacy and Identity Management for Europe, Project Leaflet. http://www.prime-project.eu.org/leaflet-pdf, Download 2004-06-01.

[Reic+02] Reichwald, R.; Meier, R.; Fremuth, N.: Die mobile Ökonomie - Definition und Spezifika. In: Reichwald, R. (Edt.) Mobile Kommunikation. Gabler: Wiesbaden, 2002, pp. 3-16.

[ReMe02] Reichwald, R.; Meier, R.: Wertschöpfungsmodelle und Wirtschaftsgüter in der mobilen Ökonomie. In: Reichwald, R. (Edt.) Mobile Kommunikation. Gabler: Wiesbaden, 2002, pp. 19-36.

[ReSc02] Reichwald, R.; Schaller, C.: M-Loyalty - Kundenbindung durch personalisierte mobile Dienste. In: Reichwald, R. (Edt.) Mobile Kommunikation. Gabler: Wiesbaden, 2002, pp. 263-286.

[Schw+02] Schweizer, L.; Meinhardt, Y.; Krys, C.; Fuest, K.: Auswirkungen der UMTSLizenzvergabe auf den Unternehmenswert und Implikationen für die Geschäftsmodelle von Mobilfunkunternehmen. In: Reichwald, R. (Edt.) Mobile Kommunikation. Gabler: Wiesbaden, 2002, pp. 85-98.

[ScLi02] Schackmann, J.; Link, H.: Intermediaries for the Provision of Mass Customized Digital Goods in Electronic Commerce. In: Rautenstrauch, C.; Seelmann-Eggebert, R.; Turowski, K. (Edt.) Moving into Mass Customization. Springer: Berlin, 2002, pp. 153172.

[Skon02] Skonnard, A.: The Birth of Web Services. http://msdn.microsoft.com/msdnmag/issues/02/10/XMLFiles/default.aspx, $\quad 2002$, Download 2004-06-07.

[Sun04] Sun: Java 2 Platform, Enterprise Edition (J2EE) Overview. http://java.sun.com/j2ee/overview.html, Download 2004-06-07.

[ThLa03] Thai, T.; Lam, H.: .Net Framework Essentials. O’Reilly \& Associates: New York, 2003.

[W3C01] W3C Consortium: Semantic Web. http://www.w3.org/2001/sw, Download 200406-07. 